\title{
Galaxy Interactions, Star Formation History, and Bulgeless Galaxies
}

\author{
Shardha Jogee ${ }^{1}$ \\ ${ }^{1}$ Department of Astronomy, University of Texas at Austin, \\ 1 University Station C1400, Austin, TX 78712-0259 \\ email: sj@astro.as.utexas.edu
}

\begin{abstract}
Hierarchical $\Lambda$ CDM models provide a successful paradigm for the growth of dark matter on large scales, but they face important challenges in predicting how the baryonic components of galaxies evolve. I present constraints on two aspects of this evolution: (1) The interaction history of galaxies over the last $7 \mathrm{Gyr}$ and the impact of interactions on their star formation properties, based on Jogee et al. (2008a,b); (2) Constraints on the origin of bulges in hierarchical models and the challenge posed in accounting for galaxies with low bulge-to-total ratios, based on Weinzirl, Jogee, Khochar, Burkert, \& Kormendy (2008, hereafter WJKBK08).
\end{abstract}

Keywords. galaxies: evolution, galaxies: interactions, galaxies: structure, galaxies: bulge

\section{Galaxy Interactions and their Star Formation over the Last $\mathbf{7}$ Gyr}

The merger history of galaxies impacts the mass assembly (e.g., Dickinson et al. 2003), star formation history, AGN activity (e.g., Springel. et al. 2005b) and structural evolution of galaxies. The merger rate/fraction at $z>1$ remains highly uncertain, owing to relatively modest volumes and bandpass shifting effects, but with a general trend towards higher merger fractions at higher redshifts Even the merger rate at $z<1$ has proved hard to robustly measure for a variety of reasons, ranging from small samples in early studies, to different methods on large samples in later studies.

In Jogee et al. (2008a,b), we have performed a complementary and comprehensive observational estimate of the frequency of interacting galaxies over $z \sim 0.24-0.80$ (lookback times of 3-7 Gyr), and the impact of interactions on the star formation (SF) of galaxies over this interval. Our study is based on HST ACS, COMBO-17, and Spitzer $24 \mu \mathrm{m}$ data from the GEMS survey. We use a large sample of $\sim 3600\left(M \geqslant 1 \times 10^{9} M_{\odot}\right)$ galaxies and $\sim 790$ high mass $\left(M \geqslant 2.5 \times 10^{10} M_{\odot}\right)$ galaxies for robust number statistics. Two independent methods are used to identify strongly interacting galaxies: a tailored visual classification system complemented with spectrophotometric redshifts and stellar masses, as well as the CAS merger criterion $(A>0.35$ and $A>S$; Conselice 2003), based on CAS asymmetry $A$ and clumpiness $S$ parameters. This allows one of the most extensive comparisons to date between CAS-based and visual classification results. We set up this visual classification system so as to target interacting systems whose morphology and other properties suggest they are a recent merger of mass ratio $M 1 / M 2>1 / 10$. While many earlier studies focused only on major mergers, we try to constrain the frequency of minor mergers as well, since they dominate the merger rates in $\Lambda$ CDM models. Some of our results are outlined below.

(1) Among 790 high mass galaxies, the fraction of visually-classified interacting systems over lookback times of 3-7 Gyr ranges from $9 \% \pm 5 \%$ at $z \sim 0.24-0.34$, to 8\% $\pm 2 \%$ at $z \sim 0.60-0.80$, as averaged over every Gyr bin.(Fig. 1a). These systems appear to be in merging or post-merger phases, and are candidates for a recent merger of mass 
ratio $M 1 / M 2>1 / 10$. Similar results on the interaction fraction are reported by Lotz et al. (2008). The lower limit on the major $(M 1 / M 2>1 / 4)$ merger fraction ranges from $1.1 \%$ to $3.5 \%$ over $z \sim 0.24-0.80$. The corresponding lower limit on the minor $(1 / 10$ $\leqslant M 1 / M 2<1 / 4)$ merger fraction ranges from $3.6 \%$ to $7.5 \%$. This is the first, albeit approximate, empirical estimate of the frequency of minor mergers over the last 7 Gyr.

(2) For an assumed value of $\sim 0.5$ Gyr for the visibility timescale, it follows that each massive $\left(M \geqslant 2.5 \times 10^{10} M_{\odot}\right)$ galaxy has undergone $\sim 0.7$ mergers of mass ratio $>1 / 10$ over the redshift interval $z \sim 0.24-0.80$. Of these, we estimate that $1 / 4$ are major mergers, $2 / 3$ are minor mergers, and the rest are ambiguous cases of major or minor mergers. The corresponding merger rate $R$ is a few $\times 10^{-4}$ galaxies $\mathrm{Gyr}^{-1} \mathrm{Mpc}^{-3}$. Among $\sim 2840$ blue cloud galaxies of mass $M \geqslant 1.0 \times 10^{9} M_{\odot}$, similar results hold.

(3) We compare our empirical merger rate $R$ for high mass $\left(M \geqslant 2.5 \times 10^{10} M_{\odot}\right)$ galaxies to predictions from different $\Lambda$ CDM-based simulations of galaxy evolution, including the halo occupation distribution (HOD) models of Hopkins et al. (2007); semianalytic models (SAMs) of Somerville et al. (2008), Bower et al. (2006), and Khochfar \& Silk (2006); and smoothed particle hydrodynamics (SPH) cosmological simulations from Maller et al. (2006). To our knowledge, such extensive comparisons have not been attempted to date, and are long overdue. We find qualitative agreement between the observations and models, with the (major+minor) merger rate from different models bracketing the observed rate, and showing a factor of five dispersion (Fig. 1b). One can now anticipate that in the near future, improvements in both the observational estimates and model predictions will start to rule out certain merger scenarios and refine our understanding of the merger history of galaxies.

(4) The idea that galaxy interactions generally enhance the star formation rate (SFR) of galaxies is well established from observations (e.g., Joseph \& Wright 1985; Kennicutt et al. 1987) and simulations (e.g., Hernquist 1989; Mihos \& Hernquist 1994, 1996; Springel, Di Matteo \& Hernquist 2005b). However, simulations cannot uniquely predict the factor by which interaction enhance the SF activity of galaxies over the last 7 Gyr, since both the SFR and properties of the remnants in simulations are highly sensitive to the stellar feedback model, the bulge-to-disk $(B / D)$ ratio, the gas mass fractions, and orbital geometry (e.g., Cox et al. 2006; di Matteo et al. 2007). Thus, empirical constraints are needed. Among $\sim 3600$ intermediate mass $\left(M \geqslant 1.0 \times 10^{9} M_{\odot}\right)$ galaxies, we find that the average SFR of visibly interacting galaxies is only modestly enhanced compared to non-interacting galaxies over $z \sim 0.24-0.80$ (Fig. 1c). This result is found for SFRs based on UV, UV+IR, and UV+stacked-IR data. This modest enhancement is consistent with the results of di Matteo et al. (2007) based on numerical simulations of several hundred galaxy collisions.

(5) The SF properties of interacting and non-interacting galaxies since $z<1$ are of great astrophysical interest, given that the cosmic SFR density is claimed to decline by a factor of 4 to 10 since $z \sim 1$ (e.g., Lilly et al. 1996; Ellis et al. 1996; Hopkins 2004; Pérez-González et al. 2005; Le Floc'h et al. 2005). We therefore set quantitative limits on the contribution of obviously interacting systems to the UV-based and UV+IR-based SFR density over $z \sim 0.24-0.80$. Among $\sim 3600$ intermediate mass $\left(M \geqslant 1.0 \times 10^{9} M_{\odot}\right)$ galaxies, we find that visibly interacting systems only account for a small fraction $(<30 \%)$ of the cosmic SFR density over lookback times of $\sim 3-7$ Gyr $(z \sim 0.24-0.80$; Fig. (1d)). Our result is consistent with that of Wolf et al. (2005) over a smaller lookback time interval of $\sim 6.2-6.8 \mathrm{Gyr}$. In effect, our result suggests that the behavior of the cosmic SFR density over the last 7 Gyr is predominantly shaped by non-interacting galaxies, rather than strongly interacting galaxies. This suggests that the observed decline in the 
cosmic SFR density since $z \sim 0.80$ is largely the result of a shutdown in the SF of non-interacting galaxies.

\section{The origin of bulges and the problem of bulgeless galaxies}

In $\Lambda$ CDM models of galaxy evolution, there are in principle three main mechanisms to build bulges of spiral galaxies: major mergers, minor mergers, and secular processes (see WJKBK08 for details). The major merger of two spiral galaxies destroys the disk component and leaves behind a classical bulge, around which a stellar disk forms when hot gas in the halo subsequently cools, settles into a disk, and forms stars. Minor mergers can also grow bulges in several ways. A tidally induced bar and/or direct tidal torques from the companion can drive gas into the inner kpc (e.g., Quinn et al. 1993; Hernquist \& Mihos 1995; Jogee 2006 and references therein), where subsequent SF forms a compact high $v / \sigma$ stellar component, or disky pseudobulge. In addition, the stellar core of the satellite can sink to the central region via dynamical friction. Finally, bulges can also have a secular origin: here, a stellar bar or globally oval structure in a non-interacting galaxy drives gas inflow into the inner $\mathrm{kpc}$, where subsequent SF forms a disky pseudobulge (e.g., Kormendy 1993; Jogee 1999; Kormendy \& Kennicutt 2004; Jogee, Scoville, \& Kenney 2005).

These different mechanisms to form bulges have been postulated for a long time. However, what is still missing is a quantitative assessment of the relative importance of different bulge formation pathways in high and low mass spirals. For instance, although bulges are an integral part of massive present-day spiral galaxies, we still cannot answer the following basic question: do most bulges in massive spirals form via major mergers, minor mergers, or secular processes?

Another thorny issue is the prevalence of bulgeless galaxies. There is rising evidence that bulgeless galaxies are quite common in the local Universe (e.g., Böker et al. 2002; Kautsch et al. 2006; BJM08a; Kormendy \& Fisher 2008). Yet, in $\Lambda$ CDM models of galaxy evolution, most galaxies that had a past major merger at a time when their mass was a fairly large fraction of their present-day mass, are expected to have a significant bulge. So far, no quantitative comparisons have been done between observations and model predictions to assess how serious is the challenge posed by bulgeless galaxies.

In WJKBK08, we attempt one of the first quantitative comparisons of the properties of bulges in a fairly complete sample of high mass $\left(M_{\star} \geqslant 1.0 \times 10^{10} M_{\odot}\right)$ spirals to predictions from $\Lambda$ CDM-based simulations of galaxy evolution. We derive the bulgeto-total mass ratio $(B / T)$ and bulge Sérsic index $n$ by performing $2 D$ bulge-disk-bar decomposition on $H$-band images of 146 bright, high mass, moderately inclined spirals.

(1) Interestingly, we find that as many as $\sim 56 \%$ of high mass spirals have low $n \leqslant 2$ bulges: such bulges exist in barred and unbarred galaxies across all Hubble types (Fig. 2a). Furthermore a striking $\sim 66 \%$ of high mass spirals have $B / T \leqslant 0.2$ (Figs. 3a and $3 \mathrm{~b}$ ).

(2) We compare the observed distribution of bulge $B / T$ in high mass spirals to predictions from $\Lambda$ CDM-based semi-analytical models. In the models, a bulge with $B / T \leqslant 0.2$ can exist in a galaxy with a past major merger, only if the last major merger occurred at $z>2$ (lookback $>10 \mathrm{Gyr}$ ). The predicted fraction of high mass spirals with a past major merger and a bulge with a present-day $B / T \leqslant 0.2$ is a factor of over fifteen smaller than the observed fraction $(\sim 66 \%)$ of high mass spirals with $B / T \leqslant 0.2$ (Fig. $2 \mathrm{~b})$. The comparisons rule out major mergers as the main formation pathway for bulges in high mass spirals. Contrary to common perception, bulges built via major mergers seriously fail to account for the bulges present in $\sim 66 \%$ of high mass spirals. 
(3) In the models, the majority of low $B / T \leqslant 0.2$ bulges exist in systems that have experienced only minor mergers, and no major mergers (Fig. 2b). These bulges can be built via minor mergers and secular processes. So far, we explored one realization of the model focusing on bulges built via satellite stars in minor mergers and find good agreement with the observations. Future models will explore more realistic minor merger scenarios and secular processes.

\section{References}

Böker, T. Laine, S., van der Marel, R. P., et al. 2002, AJ, 123, 1389

Bower, R. G., Benson, A. J., Malbon, R., et al. 2006, MNRAS, 370, 645

Conselice, C. J. 2003, ApJs, 147, 1

Cox, T. J., Jonsson, P., Primack, J. R., \& Somerville, R. S. 2006, MNRAS, 373, 1013

Dickinson, M., Papovich, C., Ferguson, H. C., \& Budavári, T. 2003, ApJ, 587, 25

Di Matteo, P., Combes, F., Melchior, A.-L., \& Semelin, B. 2007, A\&A, 468, 6

Hernquist, L. 1989, Nature, 340, 687

Hernquist, L. \& Mihos, J. C. 1995, ApJ, 448, 41

Hopkins et al. 2007, ApJ, submitted (arXiv:0706.1243)

Jogee, S. 1999, Ph.D. thesis, Yale University

Jogee, S., Shlosman, I., Laine, S., et al. 2002, ApJ, 575, 156

Jogee, S., Scoville, N., \& Kenney, J. D. P. 2005, ApJ, 630, 837

Jogee, S. 2006, in Physics of Active Galactic Nuclei at all Scales, ed. D. Alloin, R. Johnson, \& P. Lira (Berlin: Springer), 143

Jogee, S. et al. 2008a, in Formation and Evolution of Galaxy Disks, ed. J. G. Funes, S. J., \& E. M. Corsini (San Francisco: ASP), in press (arXiv:0802.3901)

Jogee, S., et al. 2008b, ApJ, submitted

Joseph, R. D. \& Wright, G. S. 1985, MNRAS, 214, 87

Kautsch, S. J., Grebel, E. K., Barazza, F. D., \& Gallagher, J. S., III 2006, A\&A, 445, 765

Kennicutt, R. C., Jr., Roettiger, K. A., Keel, W. C., van der Hulst, J. M., \& Hummel, E. 1987, AJ, 93, 1011

Khochfar, S. \& Burkert, A. 2005, MNRAS, 359, 1379

Khochfar, S., \& Silk, J. 2006, MNRAS, 370, 902

Kormendy, J. 1993, in IAU Symposium 153, Galactic Bulges, ed. H. Dejonghe \& H. J. Habing (Dordrecht: Kluwer), 209

Kormendy, J. \& Kennicutt, R. C. 2004, ARAA, 42, 603

Le Floc'h, E., et al. 2005, ApJ, 632, 169

Lilly, S. J., Le Fevre, O., Hammer, F., \& Crampton, D. 1996, ApJl, 460, L1

Lotz, J. M., et al. 2008, ApJ, 672, 177

Maller, A. H., Katz, N., Kereš, D., Davé, R., \& Weinberg, D. H. 2006, ApJ, 647, 763

Mihos, J. C. \& Hernquist, L. 1994, ApJ, 437, 611

Mihos, J. C. \& Hernquist, L. 1996, ApJ, 464, 641

Navarro, J. F. \& Steinmetz, M. 2000, ApJ, 538, 477

Pérez-González, P. G., et al. 2005, ApJ, 630, 8

Quinn, P. J., Hernquist, L., \& Fullagar, D. P. 1993, ApJ, 403, 74

Rix, H., et al. 2004, ApJs, 152, 163

Somerville, R. S., Hopkins, P. F., Cox, T. J., et al. 2008, MNRAS, accepted

Somerville, R. S. \& Primack, J. R. 1999, MNRAS, 310, 1087

Springel, V. \& Hernquist, L. 2005, ApJl, 622, L9

Springel, V., et al. 2005a, Nature, 435, 629

Springel, V., Di Matteo, T., \& Hernquist, L. 2005b, MNRAS, 361, 776

Steinmetz, M. \& Navarro, J. F. 2002, NewA, 7, 155

Weinzirl, T., Jogee, S., Khochfar, S., Burkert, A., \& Kormendy, J. 2008, ApJ, submitted (arXiv:0807.0040; WJKBK08)

Wolf, C., et al. 2005, ApJ, 630, 771 

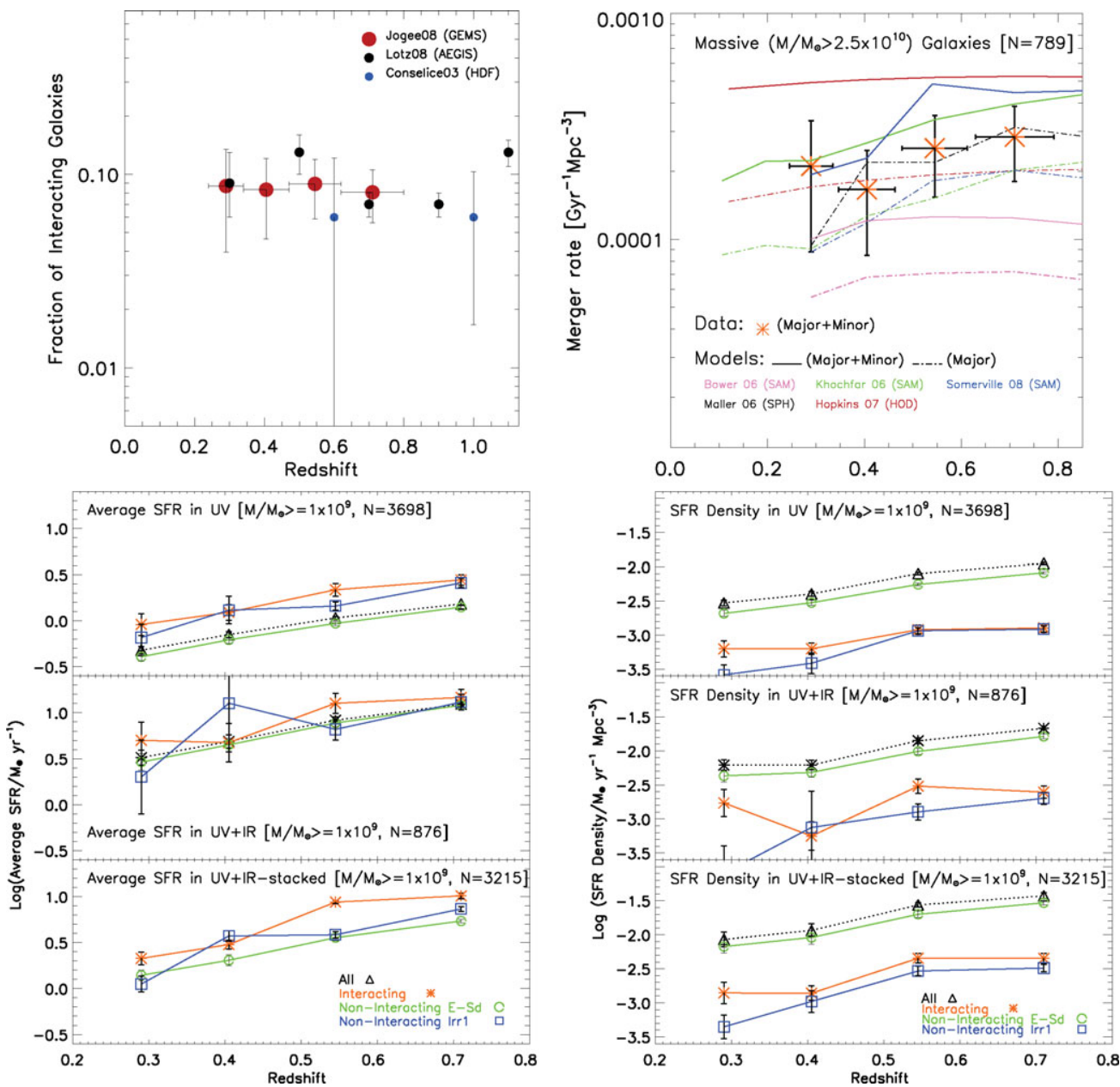

Fig. 1a (Top Left): We show the observed fraction of interacting/merging galaxies from Lotz et al. (2008), Jogee et al. (2008b), and Conselice (2003). Fig. 1b (Top Right): The empirical rate of galaxy mergers with mass ratio $M 1 / M 2>1 / 10$ (orange stars) among high mass galaxies is compared to the rate of (major+minor) mergers (solid lines) predicted by different $\Lambda$ CDM-based models of galaxy evolution. Fig. 1c (Lower Left): The average SFR of interacting and non-interacting galaxies are compared. The average UVbased SFR (top panel; based on 3698 galaxies), average UV+IR-based SFR (middle panel; based on only the 876 galaxies with 24 um detections), and average UV+IR-stacked SFR (based on 3215 galaxies with 24um coverage) are shown. In all there cases, the average SFR of interacting galaxies is only modestly enhanced compared to non-interacting E-Sd galaxies over $z \sim 0.24-0.80$ (lookback time $\sim 3-7$ Gyr). Fig. 1d (Lower Right): As in $1 \mathrm{c}$, but now showing the SFR density of galaxies. In all bins, interacting galaxies only contribute a small fraction (typically below 30\%) of the total SFR density. [All figures are from Jogee et al. (2008b)] 

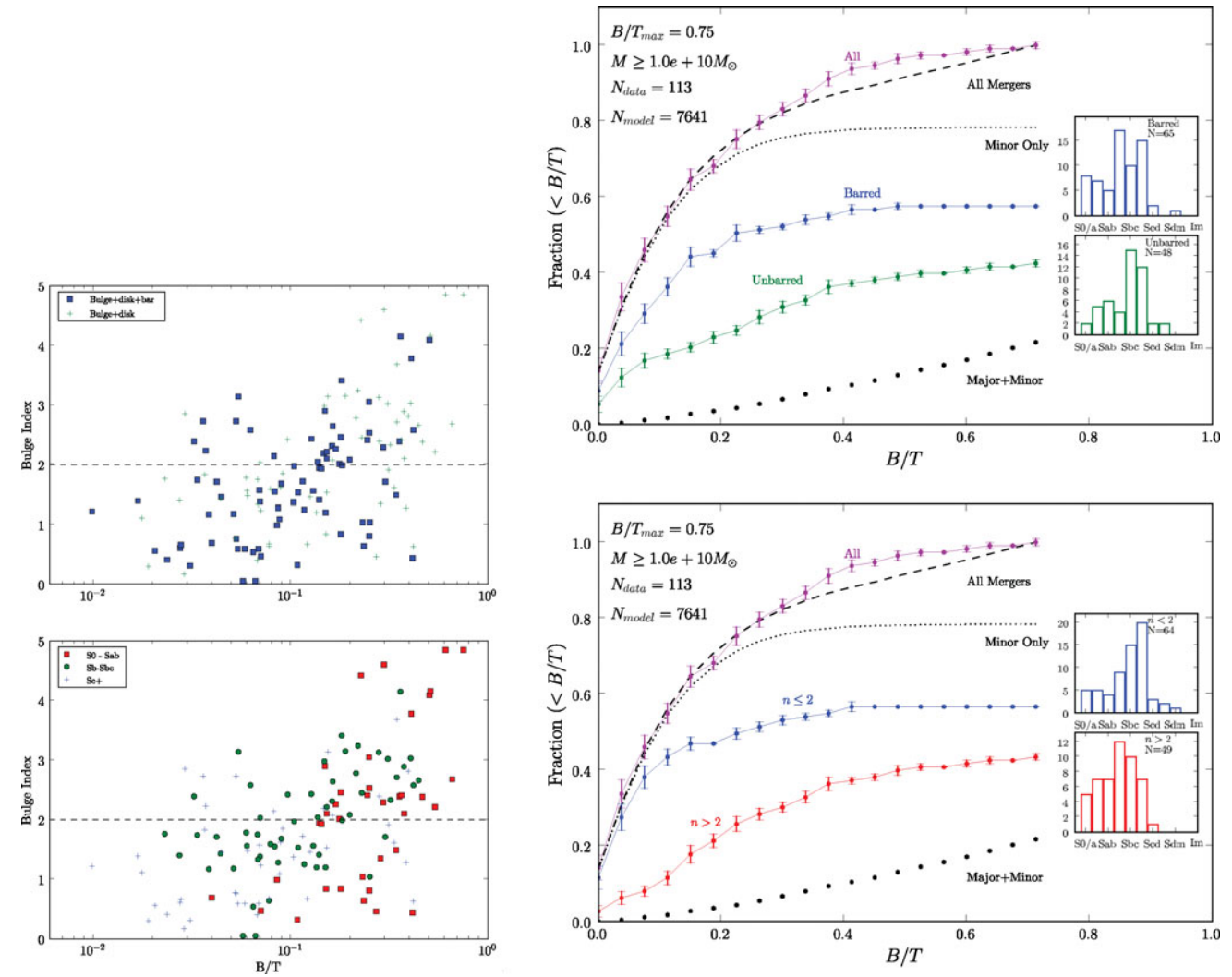

Fig. 2a (Left): The relation between $B / T$ and bulge index is shown. The legend indicates the type of decomposition used for each data point. Note that as many as $60 \%$ of bright spirals have low $n \leqslant 2$ bulges: such bulges exist in barred and unbarred galaxies across all Hubble types, and their $B / T$ ranges from 0.01 to 0.4 , with most having $B / T \leqslant 0.2$. Fig. 2 b (Right): For high mass $\left(M_{\star} \geqslant 1.0 \times 10^{10} M_{\odot}\right)$ spirals, we compare the empirical distribution of bulge-to-total mass ratio $(B / T)$ to predictions from $\Lambda C D M-b a s e d$ simulations of galaxy evolution. The y-axis shows the cumulative fraction $F$ of galaxies with $B / T \leqslant$ a given value. The magenta line shows $F$ from the data, while the other two colored lines break this $F$ in terms of bar class (top panel) or bulge $n$ (lower panel). The black dashed line shows $F$ from all model galaxies, while the black dotted line and black dots show the contribution of model galaxies that experienced, respectively, only past minor mergers and both major and minor mergers. In the models, the fraction $(\sim 3 \%)$ of high mass spirals, which have undergone a past major merger and host a bulge with $B / T \leqslant 0.2$ is a factor of over 15 smaller than the observed fraction $(\sim 66 \%)$ of high mass spirals with $B / T \leqslant 0.2$. Thus, bulges built via major mergers seriously fail to account for most of the low $B / T \leqslant 0.2$ bulges present in $\sim 66 \%$ high mass spirals. [All figures are from Weinzirl, Jogee, Khochar, Burkert, \& Kormendy (2008)] 